\title{
Interactive effects of exercise and sleep on frailty severity in community-dwelling older adults: a cross-sectional study
}

\author{
Takuya Umehara ${ }^{1}$, Akinori Kaneguchi ${ }^{1}$, Takahiro Yamasaki ${ }^{1}, 2$, Akihiro Matsuura ${ }^{1}$, \\ Nobuhiro Kito ${ }^{1,2}$, Hideki Tanaka ${ }^{2,3}$, and Kaoru Yamaoka ${ }^{1,2}$ \\ ${ }^{I}$ Department of Rehabilitation, Faculty of Rehabilitation, Hiroshima International University, Japan \\ ${ }^{2}$ Well-being and Wellness Center, Hiroshima International University, Japan \\ ${ }^{3}$ Department of Psychology, Faculty of Health Science, Hiroshima International University, Japan
}

\begin{abstract}
Objectives: This study examined the effects of the interaction between exercise and sleep on frailty severity in communitydwelling older adults.

Materials and Methods: This was a cross-sectional study. Data were collected in July 2019. In total, 2021 adults participated who responded to a questionnaire. Among them, 672 participants (317 men and 355 women) with valid responses were included in the analysis. Ordinal logistic regression analysis was performed to examine the association between frailty severity and the interaction between exercise and sleep. The dependent variable represents three different levels of frailty. The independent variables included basic information and interaction between exercise and sleep.

Results: The results of ordinal logistic regression analysis (odds ratio [OR]) showed that the period from the start of exercise $(\mathrm{OR}=0.96)$, age $(\mathrm{OR}=1.00$ for participants in their $60 \mathrm{~s}, \mathrm{OR}=1.65$ for those in their $70 \mathrm{~s}$, and $\mathrm{OR}=3.13$ for those aged $>80$ years $)$, poor subjective health perception $(\mathrm{OR}=2.12)$, poor quality of sleep $(\mathrm{OR}=1.88)$, stress $(\mathrm{OR}=1.62)$, and exercise-sleep interaction $(\mathrm{OR}=1.00$ based on good-exercise-good-sleep interaction, $\mathrm{OR}=3.09$ poor-exercise-good-sleep interaction, and $\mathrm{OR}=3.50$ poor-exercise-poorsleep interaction) significantly contributed to the model. The Nagelkerke coefficient of determination adjusted for degrees-offreedom $\left(\mathrm{R}^{2}\right)$, which represents the contribution rate of the regression equation, was 0.334 .

Conclusions: Our results suggest that a combination of good exercise and good sleep is needed to prevent frailty progression in community-dwelling older adults.
\end{abstract}

Key words: community-dwelling older adults, pre-frailty, frailty, severity, exercise-sleep interaction

\section{Introduction}

Frailty leads to adverse outcomes, including restricted mobility, reduced self-reliance and disability, falls, hospitalization, and increased mortality ${ }^{1,22}$. Frailty is a public

Received: August 10, 2021

Accepted: October 2, 2021

Correspondence: Takuya Umehara, Department of Rehabilitation, Faculty of Rehabilitation, Hiroshima International University, 55536 Kurose-Gakuendai, Higashi-Hiroshima, Hiroshima 739-2695, Japan

E-mail: start.ume0421@gmail.com

This is an open-access article distributed under the terms of the Creative Commons Attribution Non-Commercial No Derivatives (by-nc-nd) License $<\mathrm{http}: / /$ creativecommons.org/ licenses/by-nc-nd/4.0/>. health problem with a prevalence of approximately $10 \%$ in the community-dwelling elderly population on worldwide data $^{3}$, and pre-frailty is the preliminary stage of frailty. Frailty and pre-frailty are reversible conditions if treated appropriately ${ }^{4}$. There are several factors associated with the prevention or improvement of pre-frailty and frailty, with habitual exercise as one of the most important factors ${ }^{5,6)}$. Theou et $a l^{7}{ }^{7}$ examined the impact of exercise on frailty in older participants in the community based on a systematic review and reported a high-quality evidence based on which exercise for 30-45 min per exercise session for prolonged periods ( $\geq 5$ months) are effective in preventing frailty. Thus, habitual exercise as part of the activities of daily living is closely related to the prevention of frailty and is extremely important for maintaining the health of the elderly ${ }^{8}$.

Habitual exercises and adequate sleep are included in the 
prevention and improvement of frailty ${ }^{9,10)}$. Thus, inadequate sleep has been shown to increase the total number of deaths and other disorders ${ }^{11)}$. In a previous study that included 1,726 community-dwelling older adults, poor quality of sleep and shortened sleep periods $(<6 \mathrm{~h})$ were associated with the severity of pre-frailty and frailty. A high-quality systematic review by Pourmotabbed et al. ${ }^{10)}$ also indicated that shortened sleep periods are associated with an increased risk of frailty. Therefore, adequate sleep $(>6 \mathrm{~h})$ periods are extremely important for preventing and improving frailty and maintaining health in community-dwelling older adults.

Several studies have reported the importance of exercise and sleep in the prevention or improvement of frailty in community-dwelling older adults ${ }^{5-11)}$. There is a relationship between exercise and sleep. For example, habitual exercise leads to better sleep ${ }^{12)}$. In addition, some reports have indicated that habitual exercise is needed for good sleep ${ }^{13}$. Thus, it is expected that there will be an interaction between exercise and sleep. However, it is not known whether exercise and sleep have an interactive effect on frailty severity. If there is an interaction between exercise and sleep, this will constitute important information for effective frailty prevention.

Therefore, this study examined the interaction between exercise and sleep and its effects on frailty severity in community-dwelling older adults.

\section{Materials and Methods}

\section{Study design}

This was a cross-sectional study. The study protocol adhered to the Declaration of Helsinki and was reviewed and approved by the ethical committee (approval number: 19-022). Information regarding participant names, date of birth, and address were not collected to maintain the anonymity of the participants. This study was performed in accordance with strengthening the reporting of observational studies in epidemiology statements.

\section{Setting}

Data were collected from July to August 2019 as the City Health Promotion Plan (phase 2), referred to as the "Iki Iki Plan". Five thousand participants over the age of 20 living in the city were randomly selected as participants. Data from the same participants were analyzed in a previous study by Matsuura et $a l^{14)}$. However, the frailty selection criteria and analyses were different from those in the previous study. Matsuura et al. ${ }^{14)}$ divided the participants into three classes: a) no-frailty, b) cognitive frailty, and c) physical frailty, and multiple logistic regression analysis was then performed. Conversely, in this study, we classified the participants into robust, pre-frailty, and frailty groups and performed ordinal logistic regression analysis to reveal the factors that influ- enced frailty severity.

\section{Participants}

A total of 2,021 participants who responded to a preset questionnaire were included in this study. Of these, only 1,636 participants with valid responses were included. Among them, 672 participants (317 men and 355 women) aged 60 years or older were included in the analysis. These participants were divided into robust, pre-frailty, and frailty groups based on the simple frailty index ${ }^{15)}$.

\section{Variables}

Basic information and information about exercise, sleep, and frailty were extracted from a self-administered questionnaire. Sex was assessed with the following question: "Please specify your sex (male or female)"; age was assessed based the question "Please specify your age $(60,70$, or over 80 years old)"; and height and weight were assessed with "Please specify your height and weight (please write a numerical value)". Body mass index (BMI) was calculated from the height and weight. The nutritional status was assessed using the question, "Do you eat three meals a day? (yes or no)", and smoking history was determined based on the responses to the question ("smoking, not smoking, or smoked in the past"). Subjective health perception was assessed with "Please specify your health status (very well, fairly well, badly, or very badly)". Responses of "very well" and "fairly well" were considered to indicate a "healthy" status. Happiness state was assessed using the question "Are you happy? (very well, fairly well, badly, or very badly)". Responses of "very well" and "fairly well" indicate "happiness". Stress states were assessed with the question, "Did you have stress (anxiety/trouble) during the past month? (tremendous, average, some, or none)". Responses of "tremendous" and "average" indicate whether participants "experienced stress (anxiety/trouble) during the month prior to their participation in this study. Their associations with neighbors were assessed with the question, "How do you have connections with neighbors? (very good, fairly good, sometimes, or almost never)". Responses of "very good" and "fairly good" indicate "connections with neighbors are good". Trust in others was assessed using the following questions based on a four-point method: "Do you think participants are in general trust worthy?", "Do you think that a lot of participants try to take advantage of others when they have an opening?", and "Do you think that in many cases, participants try to be helpful to others? (Do not think at all, do not think much, I think, or I think very well)". The median of the total score was calculated, and the participants were classified into "good trust in others" (score was higher than the median) and "poor trust in others" (score was lower than the median) groups.

Habitual exercise was assessed with "Do you exercise? 
(yes or no)". If they did not, the exercise time and duration were scored as zero. If they performed exercises, they were considered good exercise and provided the time and duration of the exercise. Time per opportunity and duration were evaluated as follows: "How long do you exercise each time you find an opportunity for exercise? (Please write a numerical value)" and "How long did the exercise last? (please write a numerical value)". The duration since the start of exercise was treated as the duration, and the year was calculated.

The sleep status and quality of sleep were assessed with the question "Were you well-rested after sleep during the past month?" and "Are you satisfied with your overall sleep quality? (very satisfied, fairly satisfied, poorly satisfied, or not satisfied at all)". The responses of "very satisfied" and "fairly satisfied", respectively, indicated that participants felt well-rested from sleep during the month prior to the study and that they received good quality of sleep.

Frailty was determined based on the frailty index ${ }^{15}$. The frailty screening index included simple yes/no questions concerning nutrition/shrinking, physical function, physical activity, forgetfulness, and emotions/exhaustion. The index consisted of five items rated as zero or one by the participants. The five items were as follows: (1) "Have you lost 2 $\mathrm{kg}$ or more during the past 6 months? Yes=1", (2) "Do you think you walk slower than before? Yes=1", (3) "Do you go for a walk at least once a week to maintain good health status? No=1", (4) "Can you recall what happened 5 min ago? $\mathrm{No}=1$ ", and (5) "In the past 2 weeks, did you feel tired without a reason? Yes $=1$ ". We defined scores of 3 or more as frail, 1 to 2 as prefrail, and 0 as robust according to the Cardiovascular Health Study criteria ${ }^{16)}$.

\section{Statistical analysis}

Characteristic data were compared using one-way analysis of variance, Kruskal-Wallis, or $\chi^{2}$ tests, as appropriate. Variables with significant differences were subjected to post-tests. Ordinal logistic regression analysis was performed to examine the association between frailty severity and the interaction between exercise and sleep. The dependent variable represented the three levels of frailty. The independent variables provided basic information on the interaction between exercise and sleep. Adjusted odds ratios (ORs) for the incidence of disability with $95 \%$ confidence intervals were estimated. When the correlation coefficients between independent variables exceeded 0.8 , highly correlated factors with the dependent variable were adopted to resolve multicollinearity. To examine the interaction between exercise and sleep, the variables were dichotomous, that is, whether they were performing exercise and whether they felt they had rested well from sleep. The interaction between exercise and sleep was related to three variables derived from the combination of the values from the two variables: good-exercise-good-sleep, good-exercise-poorsleep, and poor-exercise-good-sleep with the fourth one, namely, poor-exercise-poor-sleep, as the reference. All statistical analyses were performed using IBM SPSS statistics package (version 23, IBM Corporation, Armonk, NY, USA).

\section{Results}

This study included 672 participants ( 317 men and 355 women) aged 60 years or older. The groups in this study were $139(20.7 \%)$ in the robust group, $418(62.2 \%)$ in the pre-frailty group, and $115(17.1 \%)$ in the frailty group. Participant characteristics are listed in Table 1. The percentages for each of the pre-frailty and frailty factors are listed in Table 2.

Ordinal logistic regression analysis was used to determine the factors that affected frailty severity. The final independent variables included age, sex, BMI, eating three meals a day, presence or absence of feelings of happiness, experience of stress during the month prior to the study, subjective health perception, smoking history, ability to connect with neighbors, trust in others, quality of sleep, and exercise-sleep interactions. The results of ordinal logistic regression analysis revealed that the period from the onset of exercise, age, subjective health perception, quality of sleep, stress, and exercise-sleep interaction were significant predictors of frailty severity (Table 3 ). The results of ordinal logistic regression analysis showed that the period from the start of exercise, age, poor subjective health perception, poor quality of sleep, stress, and exercise-sleep interaction significantly contributed to the model (Table 4). The Nagelkerke coefficient of determination adjusted for $\mathrm{R}^{2}$, which represents the contribution rate of the regression equation, was 0.334 .

\section{Discussion}

In this study, the percentages of participants who were prefrail or frail were $62.2 \%$ and $17.1 \%$, respectively. Several studies ${ }^{17,18)}$ examined the prevalence of pre-frailty and frailty based on the Japan Cardiovascular Health Study or Cardiovascular Health Study criteria and reported that the prevalence of pre-frailty and frailty were in the ranges of $51.9-65.2 \%$ and $8.3-11.2 \%$ in community-dwelling older adults, respectively. Therefore, the health status of the participants included in our study was similar or slightly lower than those of community-dwelling older adults in previous studies $^{17,18)}$. In this study, the prevalence values in the cases of no habitual exercise (low activity level), which is one of the factors of frailty, were $56.5 \%$ and $92.1 \%$ for participants with pre-frailty and frailty, respectively. A systematic review that investigated the prevalence of each frailty item reported that in community-dwelling older adults aged ap- 
Table 1 Self-administered questionnaire

Please specify your sex (male or female).

1. Male, 2. female

Please specify your age $(60,70$, or over 80 years old).

1. $60 \mathrm{~s}, 2.70 \mathrm{~s}, 3$. Over $80 \mathrm{~s}$

Please specify your height and weight (please write a numerical value).

Do you eat three meals a day?

1. Yes, 2. No

Do you smoke?

1. Smoking, 2. Not smoking, 3. Smoking in the past

Please specify your health status.

1. Very well, 2. Fairly well, 3. Badly, 4. Very badly

Are you happy?

1. Very well, 2. Fairly well, 3. Badly, 4. Very badly

Did you have stress (anxiety/trouble) during past month?

1. Tremendous, 2. Average, 3. Some, 4. None

How do you have connections with neighbors?

1. Very good, 2. Fairly good, 3. Sometimes, 4 . Almost never

Trust in others

1) Do you think participants are in general trust worthy?

2) Do you think that a lot of participants try to take advantage of others when they have an opening?

3) Do you think that in many cases, participants try to be helpful to others?

1. Do not think at all, 2. Do not think much, 3. I think, 4. I think very well

Do you exercise?

1. Yes, 2. No

How long do you exercise each time you find an opportunity for exercise? (please write a numerical value)

How long did the exercise last? (please write a numerical value)

Were you well-rested after sleep during the past month?

1. Very satisfied, 2. Fairly satisfied, 3. Poorly satisfied, 4. Not satisfied at all

Are you satisfied with your overall sleep quality?

1. Very satisfied, 2. Fairly satisfied, 3. Poorly satisfied, 4. Not satisfied at all

proximately 75 years, the prevalence of no habitual exercise ranged from $12.7 \%$ to $67.0 \%{ }^{17}$. Comparing the findings of these previous studies with the community-dwelling older adults in this study demonstrated that the lack of habitual exercise constitutes a major contributory factor for the onset of frailty.

The results of ordinal logistic regression analysis revealed that the period from the onset of exercise, age, subjective health perception, quality of sleep, stress, and exercise-sleep interaction were significant predictors of frailty severity. Previous studies ${ }^{5,7,9,19-28)}$ also reported that the period from the onset of exercise, age, subjective health perception, quality of sleep, and stress were associated with frailty. Therefore, our results support those of previous studies. Age is known to positively correlate with frailty severity ${ }^{19)}$. Kojima et al. ${ }^{19)}$ investigated the prevalence of frailty based on age and reported an increased frailty rate for those in their late 70s. In our study, the prevalence of frailty increased with age. Compared with participants in their $60 \mathrm{~s}$, the prevalence of frailty in participants in the 70s and 80 s was 1.65 and 3.13 times higher, respectively. BMI is also related to frailty, as demonstrated in previous reports ${ }^{20,21)}$. A longterm observational study ${ }^{20,21)}$ of men from 1970 to 2007 examined the relationship between long-term changes in BMI in four groups: 1) no change in normal range, 2) consistent overweight, 3) weight gain, and 4) weight loss and the appearance of frailty. The results showed that weight loss presents a risk of developing frailty ${ }^{20)}$. Conversely, there have been reports on the association between obesity (high BMI) and frailty, and many reports have shown that the association between BMI and frailty is U-shaped ${ }^{21)}$. In the present study, there was an effect on frailty severity at high BMI levels. Regarding subjective health perception, it is known that high subjective health perceptions are associated with high 
Table 2 Patient characteristics

\begin{tabular}{|c|c|c|c|c|c|c|c|}
\hline Variable & Category & $\begin{array}{c}\text { ALL } \\
(n=672)\end{array}$ & $\begin{array}{l}\text { Robust } \\
\text { group } \\
(\mathrm{n}=139)\end{array}$ & $\begin{array}{c}\text { Pre-frailty } \\
\text { group } \\
(\mathrm{n}=418)\end{array}$ & $\begin{array}{l}\text { Frailty } \\
\text { group } \\
(\mathrm{n}=115)\end{array}$ & $P$ value & Post-test \\
\hline Age (years) & $60 \mathrm{~s} / 70 \mathrm{~s} /$ over $80 \mathrm{~s}$ & $278 / 293 / 101$ & $67 / 56 / 16$ & $170 / 192 / 56$ & $41 / 45 / 29$ & 0.01 & b), c) \\
\hline Sex & Male/Female & $317 / 355$ & $76 / 63$ & $192 / 226$ & $49 / 66$ & 0.72 & \\
\hline $\operatorname{BMI}\left(\mathrm{kg} / \mathrm{m}^{2}\right)$ & & $22.9 \pm 3.2$ & $23.9 \pm 20.8$ & $23.0 \pm 3.2$ & $23.5 \pm 3.5$ & 0.01 & a), b) \\
\hline History of smoking & Smoking/Smoking in the past/Not smoking & $49 / 145 / 478$ & $11 / 29 / 99$ & $31 / 93 / 294$ & $7 / 23 / 85$ & 0.48 & \\
\hline Subjective health perception & Very well and fairly well/badly and very badly & $532 / 140$ & $129 / 10$ & $338 / 80$ & $65 / 50$ & $<0.01$ & a), b), c) \\
\hline Happiness & Very well and fairly well/badly and very badly & $610 / 62$ & $134 / 5$ & $382 / 36$ & $94 / 21$ & $<0.01$ & b), c) \\
\hline Stress during past 1 month & Tremendous and average/some and none & $380 / 292$ & $63 / 76$ & $232 / 186$ & $85 / 30$ & $<0.01$ & b), c) \\
\hline Connect with neighbor & $\begin{array}{l}\text { Very goodl and fairly good/sometimes and almost } \\
\text { never }\end{array}$ & $626 / 46$ & $136 / 3$ & $392 / 26$ & $98 / 17$ & $<0.01$ & b) \\
\hline Habitual exercise & Yes/No & $377 / 295$ & $123 / 16$ & $217 / 201$ & $37 / 78$ & $<0.01$ & a), b), c) \\
\hline Time per opportunity for exercise (hours) & & $0.7 \pm 1.1$ & $1.2 \pm 1.5$ & $0.6 \pm 0.9$ & $0.3 \pm 0.6$ & $<0.01$ & a), b), c) \\
\hline Period from exercise start (years) & & $6.5 \pm 10.7$ & $14.1 \pm 14.5$ & $5.1 \pm 8.8$ & $2.2 \pm 5.6$ & $<0.01$ & a), b), c) \\
\hline Rest from sleep during past 1 month & $\begin{array}{l}\text { Very satisfied and fairly satisfied/poorly satisfied } \\
\text { and not satisfied at all }\end{array}$ & $544 / 128$ & $124 / 15$ & $344 / 74$ & $76 / 39$ & $<0.01$ & b), c) \\
\hline Quality of sleep & $\begin{array}{l}\text { Very satisfied and fairly satisfied/poorly satisfied } \\
\text { and not satisfied at all }\end{array}$ & $278 / 394$ & $78 / 61$ & $175 / 243$ & $25 / 90$ & $<0.01$ & a), b), c) \\
\hline Good exercise - good sleep interaction & & 312 & 111 & 177 & 24 & $<0.01$ & a), b), c) \\
\hline Good exercise - poor sleep interaction & & 65 & 12 & 40 & 13 & & \\
\hline Poor exercise - good sleep interaction & & 232 & 13 & 167 & 52 & & \\
\hline Poor exercise - poor sleep interaction & & 63 & 3 & 34 & 26 & & \\
\hline
\end{tabular}

Post-test: a) robust group vs. pre-frailty group, b) robust group vs. frailty group, c) pre frailty group vs. frailty group. BMI: body mass index.

levels of activity ${ }^{22)}$ and decreased loneliness ${ }^{23}$. Therefore, it is presumed that high subjective health perceptions are associated with increased activity and prevention of frailty. Stress has generally been shown to increase vulnerability with advancing age. Frailty is defined as "a state of reduced resilience to stress due to the decline in reserve capacity associated with aging"). Stress is known to affect the severity of frailty. In fact, the results of this study also show that the proportion of participants with stress increases with frailty severity. Sleep quality has also been reported to be related to frailty ${ }^{9}$. Several possible underlying mechanisms may help explain the association between sleep disturbances and frailty. (1) Sleep symptoms may be markers of comorbidities and poor health status that increase the likelihood of frailty ${ }^{24}$. Although we adjusted for multiple disease covariates in the present study, we could not exclude this possibility. (2) Disrupted circadian rhythms induced by sleep disorders may contribute to the dysregulation of the immune system with increased systemic inflammatory factors that contribute to the development of frailty ${ }^{25}$. (3) Sleep disorders may contribute to increased oxidative stress levels and alterations in metabolic pathways favoring catabolism, which could serve as a combined risk for the development of frailty ${ }^{26}$. (4) Dis- turbed sleep patterns may reduce growth hormone, insulinlike growth factor-1, and sex hormones (such as testosterone secretion) levels, which in turn enhance muscle proteolysis, thus leading to sarcopenia and frailty ${ }^{27}$. Regarding the exercise period, longer periods from the onset of exercise were associated with lower frailty severity ${ }^{7}$. Accordingly, the period from the onset of exercise decreased with frailty severity in this study. In addition to the duration of exercise, other factors, such as frequency and content, are involved in preventing frailty severity. For example, high frequency of exercise $^{5}$ and exercise, including resistance training ${ }^{28}$, are associated with the exacerbation of frailty severity.

In the present study, poor-exercise-poor-sleep interaction yielded a 3.5-fold higher OR in frailty compared with OR values attributed to good-exercise-good-sleep interactions. In other words, participants with good-exercisegood-sleep patterns had a lower risk of frailty than those with poor-exercise-poor-sleep patterns. The odds ratio of poor exercise to good sleep interaction was 3.09, indicating that exercise had a strong influence on the severity of frailty. The reason for this may be related to the fact that this study used the frailty index, which reflects physical frailty, and we did not assess sleep in detail. As noted in the Introduction, 
Table 3 Percentage of each factor of frailty in pre-frailty and frailty participants

\begin{tabular}{lccc}
\hline & Pre-frailty group $(\mathrm{n}=418)$ & Frailty group $(\mathrm{n}=115)$ & $P$ value \\
\hline Weight loss (\%) & 8.1 & 41.7 & $<0.01$ \\
Exhaustion (\%) & 17.5 & 78.3 & $<0.01$ \\
Low memory (\%) & 5.7 & 27.8 & $<0.01$ \\
Slow gait speed (\%) & 57.2 & 93.9 & $<0.01$ \\
Low activity level (\%) & 56.5 & 92.1 & $<0.01$ \\
\hline
\end{tabular}

Table 4 Ordinal logistic regeression analysis

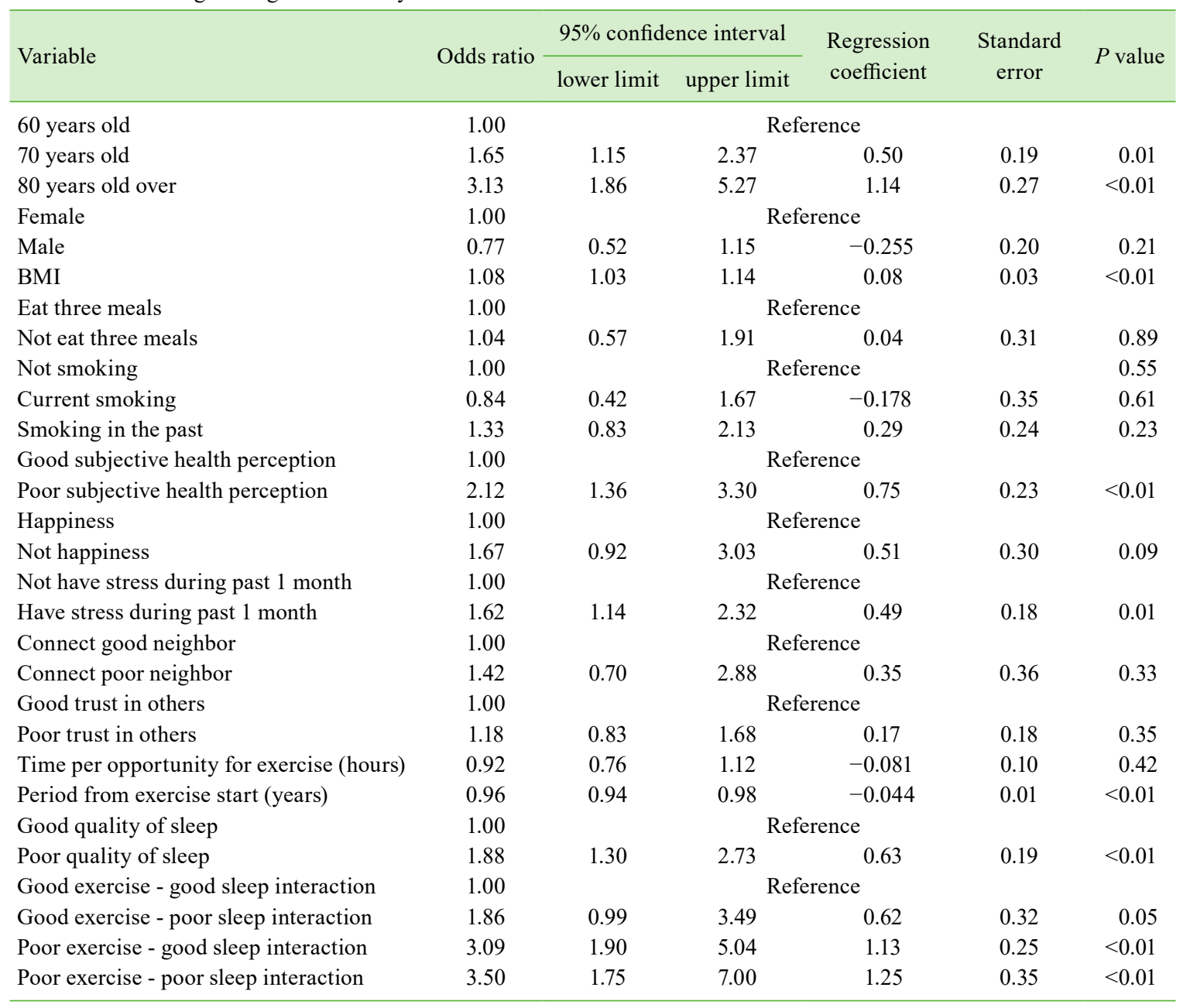

BMI: body mass index. Model: $\chi^{2} P<0.05$, Nagelkerke $\mathrm{R}^{2}=0.334$.

a relationship exists between exercise and sleep, and good sleep is essential for good exercise ${ }^{12)}$. Moreover, the OR value increased if sleep was good (3.09 vs. 3.50). Therefore, we consider that the combination of good exercise and good sleep is more effective than good exercise alone. Several studies have reported the effects of exercise or sleep alone on frailty, but the interaction between exercise and sleep has not been examined. To the best of our knowledge, this is the first study to report an interactive effect of exercise and sleep on frailty severity. Multiple factors, including inflam- mation, oxidative stress, and decreased levels of anabolic hormones, contribute to the development of frailty ${ }^{29-31}$. As mentioned above, sleep disturbances negatively affect inflammation, oxidative stress, and anabolic hormones. Conversely, habitual exercise had a positive effect on these factors. Habitual exercise decreased the markers of inflammation, such as interleukin-6 (IL-6) and C-reactive protein, and increased activity was associated with higher levels of anti-inflammatory mediators, such as adiponectin and IL$10^{29)}$. Chronic exercise also reduces oxidative stress associ- 
ated with aging ${ }^{29}$. Additionally, prolonged exercise may improve the levels of an anabolic hormone, insulin-like growth factor- ${ }^{29)}$. Thus, although habitual exercises or good-sleep patterns alone may reduce the risk of frailty, the combination of these two factors will have a synergistic effect and further reduce the risk of frailty.

The three limitations of this study are as follows. First, mental frailty was not considered in this study. There are three aspects of frailty: physical, social, and mental. A previous study ${ }^{32}$ reported a worse prognosis for multifaceted frailty, including physical, mental, and social frailty, compared with physical frailty alone. Therefore, future studies are needed to develop models to predict improvements attributed to multifaceted frailty, including mental frailty. Second, the relationship between disease severity and frailty severity was not considered in this study. We were unable to investigate the presence of the disease. These diseases have been found to influence frailty severity ${ }^{33}$. Future prospective studies should examine the interactions between the disease and sleep. Thirdly, the assessment of sleep was rough. Sleep can be assessed in several ways, including the Pittsburgh Sleep Quality Index, sleep duration, sleep wartime, and number of mid-wake awakenings. However, this study was conducted using a questionnaire because it was a large study. Therefore, it is difficult to evaluate sleep in detail. In the future, a detailed evaluation of sleep and its interaction with exercise should be conducted.

\section{Conclusion}

Our results suggest that a combination of good exercise and good sleep is needed to prevent the exacerbation of frailty in community-dwelling older adults.

\section{Acknowledgement}

We thank Kimihiko Tokumori, Ph.D. (Department of Rehabilitation, Faculty of Rehabilitation, Hiroshima International University and Well-Being and Wellness Center, Hiroshima International University) and Kanako Yamauchi, Ph.D. (Department of Psychology, Faculty of Health Science, Hiroshima International University) for their advice regarding the data collection method, and Masayuki Kakehashi, Ph.D. (Department of Health Informatics, Graduate School of Biomedical and Health Sciences, Hiroshima University) for his advice regarding the statistical analysis method.

\section{References}

1. Clegg A, Young J, Iliffe S, et al. Frailty in elderly people. Lancet 2013; 381: 752-762. [Medline] [CrossRef]

2. Blodgett JM, Theou $\mathrm{O}$, Howlett SE, et al. A frailty index based on laboratory deficits in community-dwelling men predicted their risk of adverse health outcomes. Age Ageing 2016; 45: 463-468. [Medline] [CrossRef]

3. Collard RM, Boter H, Schoevers RA, et al. Prevalence of frailty in community-dwelling older persons: a systematic review. J Am Geriatr Soc 2012; 60 : 1487-1492. [Medline] [CrossRef]

4. Yoshimura Y, Wakabayashi H, Yamada M, et al. Interventions for treating sarcopenia: a systematic review and meta-analysis of randomized controlled studies. J Am Med Dir Assoc 2017; 18: 553.e1-553.e16. [Medline] [CrossRef]

5. Apóstolo J, Cooke R, Bobrowicz-Campos E, et al. Effectiveness of interventions to prevent pre-frailty and frailty progression in older adults: a systematic review. JBI Database Syst Rev Implement Reports 2018; 16: 140-232. [Medline] [CrossRef]

6. Tanimura C, Matsumoto H, Tokushima Y, et al. Self-care agency, lifestyle, and physical condition predict future frailty in community-dwelling older people. Nurs Health Sci 2018; 20: 31-38. [Medline] [CrossRef]

7. Theou O, Stathokostas L, Roland KP, et al. The effectiveness of exercise interventions for the management of frailty: a systematic review. J Aging Res 2011 ; 2011: 569194. [Medline] [CrossRef]

8. Booth FW, Roberts CK, Laye MJ. Lack of exercise is a major cause of chronic diseases. Compr Physiol 2012; 2: 1143-1211. [Medline] [CrossRef]

9. Sun XH, Ma T, Yao S, et al. Associations of sleep quality and sleep duration with frailty and pre-frailty in an elderly population Rugao longevity and ageing study. BMC Geriatr 2020; 20: 9. [Medline] [CrossRef]

10. Pourmotabbed A, Boozari B, Babaei A, et al. Sleep and frailty risk: a systematic review and meta-analysis. Sleep Breath 2020; 24: 1187-1197. [Medline] [CrossRef]

11. Tamakoshi A, Ohno Y. JACC Study Group Self-reported sleep duration as a predictor of all-cause mortality: results from the JACC study, Japan. Sleep 2004; 27: 51-54. [Medline]

12. Hasan J, Urponen H, Vuori I, et al. Exercise habits and sleep in a middle-aged Finnish population. Acta Physiol Scand Suppl 1988; 574: 33-35. [Medline]

13. Shapiro CM, Bachmayer D. Epidemiological aspects of sleep in general public and hospital outpatient samples. Acta Physiol Scand Suppl 1988; 574: 41-43. [Medline]

14. Matsuura A, Umehara T, Yamasaki T, et al. Factors affecting cognitive frailty in the community-dwelling older adults by questionnaire survey. J Med Eng 2021; 15: 9-15 (in Japanese).

15. Yamada M, Arai H. Predictive value of frailty scores for healthy life expectancy in community-dwelling older Japanese adults. J Am Med Dir Assoc 2015; 16: 1002.e7-1002.e11. [Medline] [CrossRef]

16. Fried LP, Tangen CM, Walston J, et al. Cardiovascular Health Study Collaborative Research Group. Frailty in older adults: evidence for a phenotype. J Gerontol A Biol Sci Med Sci 2001; 56: M146-M156. [Medline] [CrossRef]

17. Satake S, Shimada H, Yamada M, et al. Prevalence of frailty among community-dwellers and outpatients in Japan as defined by the Japanese version of the 
Cardiovascular Health Study criteria. Geriatr Gerontol Int 2017; 17: 2629-2634. [Medline] [CrossRef]

18. Shirooka H, Nishiguchi S, Fukutani N, et al. Cognitive impairment is associated with the absence of fear of falling in community-dwelling frail older adults. Geriatr Gerontol Int 2017; 17: 232-238. [Medline] [CrossRef]

19. Kojima G, Iliffe S, Taniguchi Y, et al. Prevalence of frailty in Japan: a systematic review and meta-analysis. J Epidemiol 2017; 27: 347-353. [Medline] [CrossRef]

20. Strandberg TE, Stenholm S, Strandberg AY, et al. The "obesity paradox", frailty, disability, and mortality in older men: a prospective, longitudinal cohort study. Am J Epidemiol 2013; 178: 1452-1460. [Medline] [CrossRef]

21. Hubbard RE, Lang IA, Llewellyn DJ, et al. Frailty, body mass index, and abdominal obesity in older people. J Gerontol A Biol Sci Med Sci 2010; 65: 377-381. [Medline] [CrossRef]

22. Maddox GL. Some correlates of differences in self-assessment of health status among the elderly. J Gerontol 1962; 17: 180-185. [Medline] [CrossRef]

23. Berkman LF, Melchior M, Chastang JF, et al. Social integration and mortality: a prospective study of French employees of Electricity of France-Gas of France: the GAZEL Cohort. Am J Epidemiol 2004; 159: 167-174. [Medline] [CrossRef]

24. Ensrud KE, Blackwell TL, Ancoli-Israel S, et al. Sleep disturbances and risk of frailty and mortality in older men. Sleep Med 2012; 13: 1217-1225. [Medline] [CrossRef]

25. Mehra R. Failing sleep? Beware of frailty or death. Sleep Med 2012; 13: 1211-1212. [Medline] [CrossRef]

26. Cochen V, Arbus C, Soto MEV, et al. Sleep disorders and their impacts on healthy, dependent, and frail older adults. J Nutr Health Aging 2009; 13: $322-329$. [Medline] [CrossRef]

27. Piovezan RD, Abucham J, Dos Santos RV, et al. The impact of sleep on age-related sarcopenia: possible connections and clinical implications. Ageing Res Rev 2015; 23(Pt B): 210-220. [Medline] [CrossRef]

28. Puts MTE, Toubasi S, Andrew MK, et al. Interventions to prevent or reduce the level of frailty in community-dwelling older adults: a scoping review of the literature and international policies. Age Ageing 2017; 46: 383-392. [Medline]

29. Angulo J, El Assar M, Álvarez-Bustos A, et al. Physical activity and exercise: strategies to manage frailty. Redox Biol 2020; 35: 101513. [Medline] [CrossRef]

30. Ma L, Chan P. Understanding the physiological links between physical frailty and cognitive decline. Aging Dis 2020; 11: 405-418. [Medline] [CrossRef]

31. Álvarez-Satta M, Berna-Erro A, Carrasco-Garcia E, et al. Relevance of oxidative stress and inflammation in frailty based on human studies and mouse models. Aging (Albany NY) 2020; 12: 9982-9999. [Medline] [CrossRef]

32. Rodríguez-Pascual C, Paredes-Galán E, Ferrero-Martínez AI, et al. The frailty syndrome is associated with adverse health outcomes in very old patients with stable heart failure: a prospective study in six Spanish hospitals. Int J Cardiol 2017; 236: 296-303. [Medline] [CrossRef]

33. Ida S, Kaneko R, Imataka K, et al. Relationship between frailty and mortality, hospitalization, and cardiovascular diseases in diabetes: a systematic review and meta-analysis. Cardiovasc Diabetol 2019; 18: 81. [Medline] [CrossRef] 